\title{
Development and Validation of a Risk Nomogram Model for Predicting Contrast-Induced Acute Kidney Injury in Patients with Non-ST-Elevation Acute Coronary Syndrome Undergoing Primary Percutaneous Coronary Intervention
}

\author{
Kai Ma $\mathbb{D}^{*}$, Jing Li*, Guoqi Shen, Di Zheng, Yongli Xuan, Yuan Lu, Wenhua Li \\ Department of Cardiology, The Affiliated Hospital of Xuzhou Medical University, Xuzhou, Jiangsu, 221004, People's Republic of China \\ *These authors contributed equally to this work \\ Correspondence: Wenhua Li, Tel +86 18052268293, Email xzwenhua0202@163.com
}

Objective: To establish a nomogram model to predict the risk of contrast-induced acute kidney injury (CI-AKI) by analyzing the risk factors of CI-AKI and to evaluate its effectiveness.

Methods: Retrospectively analyze the clinical data of non-ST-elevation acute coronary syndrome (NSTE-ACS) patients who underwent percutaneous coronary intervention (PCI) in our cardiology department from September 2018 to June 2021. Of these, patients who underwent PCI in an earlier period formed the training cohort (70\%; $n=809)$ for nomogram development, and those who underwent PCI thereafter formed the validation cohort $(30 \% ; \mathrm{n}=347)$ to confirm the model's performance. The independent risk factors of CI-AKI were determined by LASSO regression and multivariable logistic regression analysis. By using R software from which nomogram models were subsequently generated. The nomogram was developed and evaluated based on discrimination, calibration, and clinical efficacy using the concordance statistic (C-statistic), calibration plot, and decision curve analysis (DCA), respectively.

Results: The nomogram consisted of six variables: age $>75$, left ventricular ejection fraction, diabetes mellitus, fibrinogen-to-albumin ratio, high-sensitive $\mathrm{C}$-reactive protein, and lymphocyte count. The $\mathrm{C}$-index of the nomogram is 0.835 (95\% CI: $0.800-0.871)$ in the training cohort and 0.767 (95\% CI: $0.711-0.824)$ in the validation cohort, respectively. The calibration plots exhibited that the nomogram was in good agreement between prediction and observation in the training and validation cohorts. Decision curve analysis and clinical impact curve suggested that the predictive nomogram had clinical utility.

Conclusion: The nomogram model established has a good degree of differentiation and accuracy, which is intuitively and individually to screen high-risk groups and has a certain predictive value for the occurrence of CI-AKI in NSTE-ACS patients after PCI.

Keywords: nomogram, risk forecasting model, non-ST-elevation acute coronary syndrome, percutaneous coronary intervention, contrast-induced acute kidney injury

\section{Introduction}

The term "contrast-induced acute kidney injury (CI-AKI)" was introduced by the Kidney Disease Improving Global Outcomes (KDIGO) working group in 2012. ${ }^{1}$ It is characterized by a decrease in renal function within a few days after intravascular injection of iodine contrast medium. CI-AKI is a common and serious complication after percutaneous coronary intervention (PCI) in patients with coronary artery disease (CAD). It has been associated with irreversible deterioration of renal function, the need for dialysis, increased hospital costs, and death. ${ }^{2-4}$ Unfortunately, few strategies are effective in preventing and treating CI-AKI. Non-ST-segment elevation acute coronary syndrome (NSTE- ACS) is 
a common type of coronary artery disease, representing approximately two-thirds of patients with ACS. ${ }^{5,6}$ Although patients presenting with NSTE-ACS appear more stable at presentation, and their long-term outcome is worse. 1-year mortality is higher for patients presenting with NSTE-ACS than ST-segment elevation myocardial infarction (STEMI). ${ }^{7}$ Moreover, the prognosis of CI-AKI in patients with NSTE-ACS is worse than that of the general population. Therefore, accurate identification of NSTE-ACS patients at increased risk of CI-AKI after PCI is essential in a clinical setting. It would be of great clinical significance for clinicians to predict whether the early intervention will be needed following PCI.

A series of risk-stratification models that incorporate patient and procedural factors have been validated in past studies. ${ }^{89}$ A classical risk score including eight variables established by Mehran et a ${ }^{10}$ in 2004 is one of the most common prediction models for CI-AKI. While this tool can be used for quality reporting and other functions, it is not very helpful because it includes certain variables that cannot be known before the procedure. Furthermore, most studies have focused on independent risk factor analysis and regression equations to make predictions. Nomogram is a statistical prediction model established based on the characteristic phenotype of the disease, which is used to predict the probability of a certain outcome event in a population with certain characteristics in the future. It has the advantage of being more intuitive, vivid, and simple compared with traditional prediction methods. ${ }^{11,12}$

This retrospective study analyzed clinical data from patients with NSTE-ACS treated with PCI in our cardiology department. Independent risk factors for CI-AKI were identified by LASSO regression screening and multivariate logistic regression analysis. ${ }^{13}$ The clinical outcomes were used to establish a predictive model for screening high-risk populations to provide more accurate guidance for the early prevention of NSTE-ACS patients.

\section{Materials and Methods}

\section{Study Population}

The overall flow diagram is shown in Figure 1. The clinical data of 1421 patients with NSTE-ACS who underwent PCI in our cardiology department from September 2018 to June 2021 were retrospectively analyzed. The diagnosis of NSTE-ACS

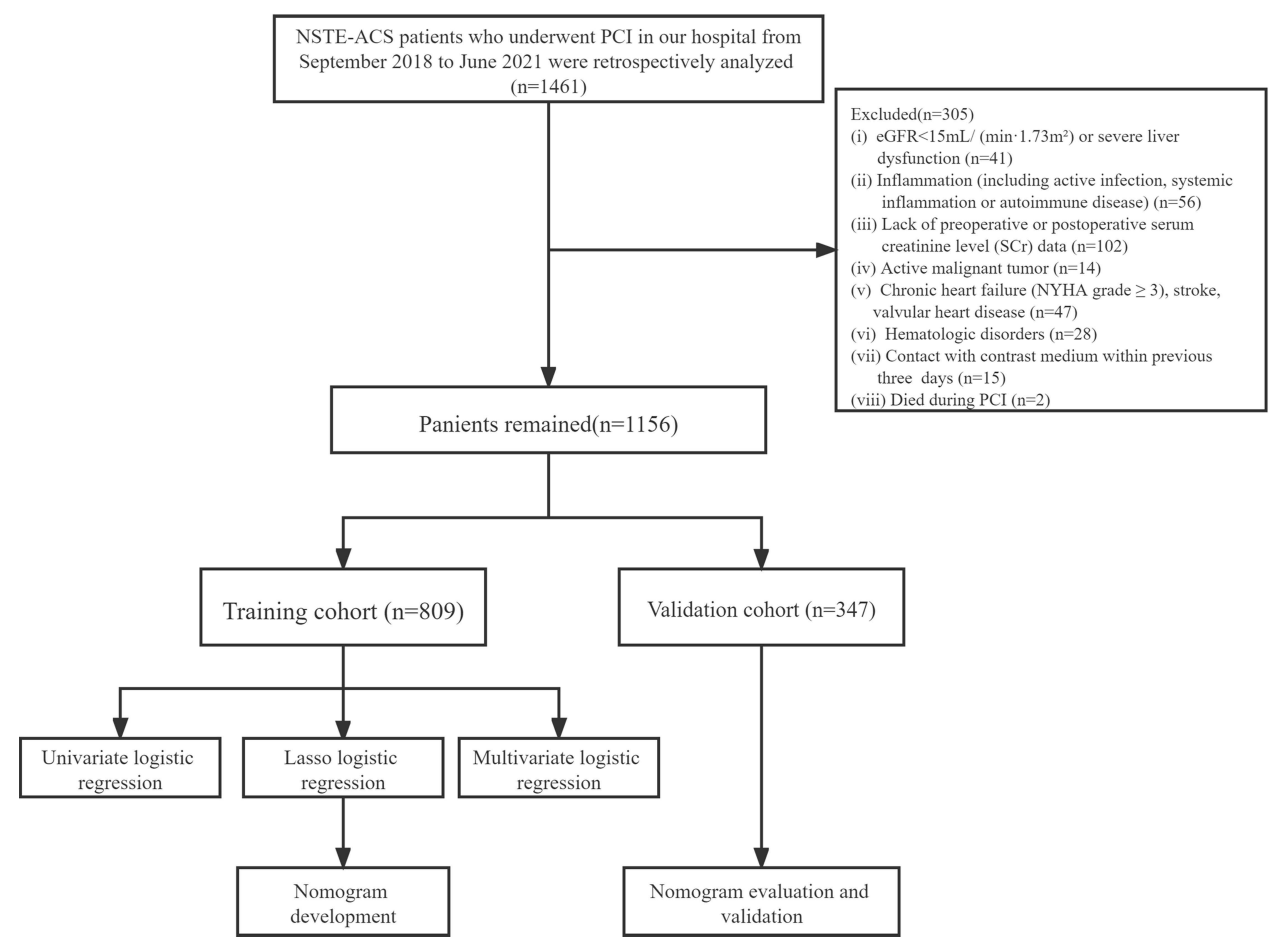

Figure I Study flow diagram.

Abbreviations: NSTE-ACS, non-ST elevation acute coronary syndrome; PCI, percutaneous transluminal coronary intervention; eGFR, estimated glomerular filtration rate; NYHA, New York Heart Association. 
was based on criteria established by the American College of Cardiology. The inclusion criteria were: (i) diagnosis of NSTEMI; (ii) dynamic or possible new serial ST-T changes indicating progressive ischemia; (iii) transient ST-segment elevation. ${ }^{14,15}$

\section{Exclusion Criteria}

The exclusion criteria were those patients with severe chronic kidney disease (defined as estimated glomerular filtration rate $(\mathrm{eGFR})<15 \mathrm{~mL} /\left(\mathrm{min} \cdot 1.73 \mathrm{~m}^{2}\right)$ ) or severe liver dysfunction, inflammation (including active infection, systemic inflammation or autoimmune disease), malignancy, chronic heart failure (New York Heart Association (NYHA) grade $\geq 3$ ), stroke, valvular heart disease, hematologic disorders, missing preoperative or postoperative serum creatinine ( $\mathrm{SCr}$ ), with exposure to a contrast medium within previous three days. Patients who died during PCI were also excluded. Finally, 1156 patients remained in the study sample, including 785 males $(67.9 \%)$. The mean age was $63.96 \pm 11.77$ years. Of these, patients who underwent PCI in an earlier period formed the training cohort (from September 2018 to October 2020, $\mathrm{n}=809$ ) for nomogram development, and those who underwent PCI thereafter formed the independent validation cohort (from November 2020 to June 2021, $n=347$ ) to confirm the model's performance.

\section{$\mathrm{PCl}$ Method}

In this study, all patients who planned to undergo PCI were routinely given dual antiplatelet therapy and 3000 IU of ordinary heparin anticoagulation before the procedure, and 100IU $/ \mathrm{kg}$ heparin could be added during PCI according to the patient's body weight. PCI was performed according to standard clinical practice using standard catheters, guidewires, balloon catheters, and stents via the radial artery approaches. Nonionic, iso-osmolar contrast medium was administered during the procedure (ioversol, Yangtze River Pharmaceutical Group), the osmotic concentration was $800 \mathrm{mOsm} / \mathrm{Kg}$. Interventional physicians determined the dose according to the patient's condition during the procedure. Furthermore, all patients received normal saline at a rate of $1 \mathrm{~mL} \cdot \mathrm{kg}^{-1} \cdot \mathrm{h}^{-1}$ before the procedure and continued for 12 hours after the procedure according to the guidelines. In patients with left ventricular ejection fraction (LVEF) less than $40 \%$ or heart failure, the hydration rate should be reduced to $0.5 \mathrm{~mL} \cdot \mathrm{kg}^{-1} \cdot \mathrm{h}^{-1} \cdot 16,17$

\section{Definition}

The European Society of Urogenital Radiology (ESUR) defines CI-AKI as: The absolute increase of serum creatinine level $\geq 0.5 \mathrm{mg} / \mathrm{dl}(44.2 \mu \mathrm{mol} / \mathrm{L})$ within $48-72$ hours after contrast medium exposure or the increase of $\geq 25 \%$ compared with the preoperative level, excluding kidney damage caused by other reasons, can be diagnosed as CI-AKI". ${ }^{18}$ Related definitions and diagnostic criteria included the following: Fibrinogen-to-albumin ratio (FAR) was defined as the concentration ratio of fibrinogen $(\mathrm{g} / \mathrm{L})$ to albumin $(\mathrm{g} / \mathrm{L})$ multiplied by $100 .{ }^{19}$ Anemia was defined as hemoglobin (HGB) concentration $<13 \mathrm{~g} / \mathrm{dL}$ for men and $<12 \mathrm{~g} / \mathrm{dL}$ for women. Chronic renal disease was considered if baseline $\mathrm{SCr}>133 \mu \mathrm{mol} / \mathrm{L}$. Hypotension was considered as the occurrence of systolic pressure $<80 \mathrm{mmHg}$ for at least 1 hour requiring inotropic agents or Intra-Aortic Balloon Pump (IABP) insertion within 24 of the PCI. The diagnostic criteria for diabetes were fasting glucose $>7 \mathrm{mmol} / \mathrm{L}$, random glucose $>11.1 \mathrm{mmol} / \mathrm{L}$, or a history of diabetes, including diet, oral medication, or insulin therapy. Current smoking is those who have smoked regularly in the past six months.

\section{Data Collection}

The baseline and clinical characteristics (including demographic data, previous medical history, vital signs on admission, laboratory indices, angiographic variables, and medication use at discharge) were gathered from the medical record systems. The venous blood samples were obtained from each participant within 24 hours of admission and at least two days after PCI at room temperature. All laboratory indices were uniformly and preoperatively performed by the laboratory of our testing center. Data collected during PCI included the target vessel (left coronary artery, left anterior descending coronary artery, left circumflex coronary artery, and right coronary artery), the total length of the implanted stent, the average diameter of the implanted stent, the number of implanted stents, contrast medium volume, and contact time. Left ventricular ejection fraction was recorded by echocardiography to evaluate left ventricular systolic function. 


\section{Statistical Analysis}

Categorical variables are expressed as counts and percentages (\%), while continuous variables are expressed as mean \pm standard deviation or median and quartile ranges. The independent sample $t$-test was used to compare the parameter values of the two groups, the Mann-Whitney $U$-test was used to compare the non-parameter values of the two groups, and the chi-square test was used to compare the classified variables. LASSO regression was used to screen out non-zero coefficient characteristics, and multivariate logistic regression was used to analyze the independent predictors of CI-AKI in NSTE-ACS patients after PCI. Based on the above factors, a predictive nomogram model of CI-AKI was constructed. We evaluated the predictive model in terms of three quantities, namely discriminative capacity, calibration ability, and clinical effectiveness. Since the consistency index (C-index) is equivalent to the area under the receiver operating characteristic curve (AUC) in logistic regression, we used the AUC to evaluate the discriminative ability of the nomogram. At the same time, Area under curve comparison between the nomogram and the Mehran Score was performed using DeLong's test. Calibration accuracy was evaluated by a calibration plot and Hosmer-Lemeshow test. Clinical effectiveness was evaluated by a decision curve analysis (DCA). All statistical analyses were performed using SPSS version 26.0 (SPSS Inc., Chicago, IL, USA), and the statistical software package R, version 4.0.3 (https://cran. r-project.org). The software packages involved in R software implementation mainly include "glmnet", "rms", "pROC", "rmda", and so on. All tests were two-tailed, and $P$ value $<0.05$ was considered statistically significant.

\section{Results}

\section{Baseline Patient Characteristics in Training Cohort}

Of the 809 patients with NSTE-ACS, 15.5\% (125/809) developed CI-AKI. However, the definition of CI-AKI is diverse, and some studies define CI-AKI as a $50 \%$ increase in serum creatinine from baseline values or $0.3 \mathrm{mg} / \mathrm{dl}$ after PCI. ${ }^{20}$ According to this calculation, the incidence of CI-AKI was $13.6 \%(110 / 809)$. There was no statistically significant difference between the two methods to calculate CI-AKI incidence $\left(\chi^{2}=1.120, P=0.290\right)$. General information and laboratory data for both groups are shown in Tables 1 and 2. Compared with the non-CI-AKI group, the CI-AKI group have a significant difference in Age $>75$ years, gender, diabetes mellitus, Anemia, some biochemical parameters (eg, glucose, albumin, fibrinogen, hemoglobin, hsCRP, lymphocyte count, neutrophil count, platelet distribution width), FAR, Neutrophil-to-Lymphocyte Ratio (NLR), LVEF, and diuretic use $(P<0.05)$.

\section{LASSO Regression and Multivariate Logistic Regression Analysis}

LASSO regression analysis showed that Age $>75$ years, gender, diabetes mellitus, anemia, diuretic use, albumin, fibrinogen, FAR, LVEF, hsCRP, hemoglobin, lymphocyte count, neutrophil count, NLR, and platelet distribution width were the more important predictors with non-zero coefficient, as shown in Figure 2. The independent predictors screened by LASSO regression were the independent variables (Age > 75 years as "yes", Age $\leq 75$ years as "no"; the presence of diabetes mellitus or hypotension as "yes" and absence as "no"; use of diuretics as "yes", "no" for non-use). Multivariate logistic regression analysis with the occurrence of CI-AKI (occurrence as "yes", non-occurrence as "no") as the dependent variable showed that Age $>75$ years, diabetes mellitus, LVEF, hsCRP, FAR, and lymphocyte count were independent predictors of the occurrence of CI-AKI after PCI in patients with NSTE-ACS (Table 3).

\section{Predictive Nomogram Development}

Based on the above analysis, this study used six variables, Age $>75$ years, diabetes, LVEF, FAR, hsCRP, and lymphocyte count, to establish a nomogram model for predicting CI-AKI after the procedure in NSTE-ACS patients. As shown in Figure 3, each of these independent predictors was projected upward to the value of the "points" at the top level of the nomogram to obtain a score within the range of 0 to 100 and then record the total score of these points, to accurately predict the risk of CI-AKI in the corresponding NSTE-ACS patients. The higher the total score, the higher the risk of CIAKI. Meanwhile, the Bootstrap internal verification method was used in this study to conduct internal verification of the nomogram model. The Hosmer-Lemeshow test result was $\chi^{2}=6.301(P=0.614)$, which proves that the model had a good degree of calibration. The Calibration curve shows a good agreement between the predicted and actual risk of CI-AKI, as 
Table I Comparison of Baseline Features and PCI Results in the Training Cohort

\begin{tabular}{|c|c|c|c|c|}
\hline Variable & $\begin{array}{c}\text { Non-CI-AKI Group } \\
(n=684)\end{array}$ & $\begin{array}{l}\text { CI-AKI Group } \\
(n=\mid 25)\end{array}$ & $\chi^{2} / t$ Value & $P$ value \\
\hline Age (year, $\bar{x} \pm s)$ & $63.90 \pm 11.88$ & $67.03 \pm 12.34$ & -2.693 & 0.007 \\
\hline Age $>75, n(\%)$ & $109(15.94)$ & $38(30.40)$ & 18.633 & $<0.001$ \\
\hline Male, n (\%) & $476(69.60)$ & $74(59.20)$ & 5.242 & 0.011 \\
\hline Hypertension, n (\%) & $358(52.34)$ & $69(55.20)$ & 0.200 & 0.527 \\
\hline Diabetes mellitus, n (\%) & $147(2 \mid .49)$ & $70(56.00)$ & 64.120 & $<0.001$ \\
\hline Smoking, n (\%) & $259(37.87)$ & $42(33.60)$ & 0.823 & 0.293 \\
\hline Systolic pressure $(\mathrm{mmHg}, \bar{x} \pm \mathrm{s})$ & $131.90 \pm 21.07$ & $130.95 \pm 22.16$ & 0.460 & 0.646 \\
\hline Diastolic pressure $(\mathrm{mmHg}, \bar{x} \pm s)$ & $78.14 \pm 13.50$ & $78.86 \pm 13.50$ & -0.550 & 0.583 \\
\hline Anemia, n (\%) & $94(13.74)$ & $27(21.60)$ & 5.130 & 0.024 \\
\hline Hypotension, n (\%) & $44(6.43)$ & $9(7.20)$ & 0.102 & 0.750 \\
\hline Intra-Aortic Balloon Pump, n (\%) & 0 & 0 & - & - \\
\hline \multicolumn{5}{|l|}{ Use of therapeutic agents, $n(\%)$} \\
\hline Aspirin, n (\%) & $684(100)$ & $125(100)$ & - & 1 \\
\hline Clopidogrel/Ticagrelor, n (\%) & $684(100)$ & $125(100)$ & - & I \\
\hline Beta-blockers, n (\%) & $566(82.75)$ & I04(83.20) & 0.003 & 0.953 \\
\hline ACEI/ARB, n (\%) & $414(60.53)$ & $72(57.60)$ & 0.401 & 0.526 \\
\hline CCB, n (\%) & $137(20.03)$ & $17(13.60)$ & 3.398 & 0.065 \\
\hline Diuretics, n (\%) & $253(36.99)$ & $72(57.60)$ & 23.146 & $<0.001$ \\
\hline Statins, n (\%) & $633(92.54)$ & $120(96.00)$ & 2.222 & 0.162 \\
\hline Nitrates, n (\%) & $47 \mid(68.86)$ & $86(68.80)$ & 0.001 & 0.979 \\
\hline \multicolumn{5}{|l|}{ Stent number per patient, n (\%) } \\
\hline Left anterior descending, $\mathrm{n}(\%)$ & $310(45.32)$ & $58(46.40)$ & 0.050 & 0.824 \\
\hline Left circumflex, n (\%) & $\mid 48(2 \mid .64)$ & $33(26.40)$ & 1.380 & 0.240 \\
\hline Right coronary artery, n (\%) & $222(32.46)$ & $32(25.60)$ & 2.306 & 0.129 \\
\hline Left main, n (\%) & $4(0.58)$ & $2(1.60)$ & 1.480 & 0.224 \\
\hline \multicolumn{5}{|l|}{ Stent number per patient, n (\%) } \\
\hline I (n, \%) & $600(87.72)$ & 104(83.20) & 1.911 & 0.167 \\
\hline$\geq 2(n, \%)$ & $84(12.28)$ & $21(16.80)$ & 1.911 & 0.167 \\
\hline Stent length $(\mathrm{mm})$ & $16.78 \pm 3.37$ & $17.84 \pm 4.02$ & 0.972 & 0.246 \\
\hline Stent diameter $(\mathrm{mm})$ & $3.21 \pm 0.35$ & $3.25 \pm 0.28$ & 1.027 & 0.312 \\
\hline Contrast medium contact time ( $\mathrm{min})$ & $35.47 \pm 17.43$ & $38.23 \pm 16.98$ & 0.921 & 0.255 \\
\hline Contrast medium> $>00(\mathrm{~mL})$ & $398(58.19)$ & $81(64.80)$ & 1.914 & 0.166 \\
\hline Mehran score & $5.25 \pm 4.25$ & $4.75 \pm 3.50$ & 1.523 & 0.214 \\
\hline
\end{tabular}

Abbreviations: $\mathrm{Cl}-\mathrm{AKI}$, contrast-induced acute kidney injury; LVEF, left ventricular ejection fraction; ACEI, angiotensin converting enzyme inhibitor; ARB, angiotensin receptor inhibitor; CCB, calcium channel blockers.

shown in Figure 4A. The C-index is $0.835(95 \% \mathrm{CI}: 0.800-0.871)$. The prediction model has a good degree of differentiation, as shown in Figure 5A. In addition, the nomogram demonstrated better predictive value among patients with NSTE-ACS while compared with Mehran Score $(Z=3.144, P=0.002$, Figure 6).

\section{Validation of the Nomogram}

In the validation cohort, there are 43 cases (12.4\%) that developed CI-AKI. The Hosmer-Lemeshow test result is $\chi^{2}=$ $4.252(P=0.834)$. The Calibration curve shows that the nomogram prediction model has a good consistency and fitting degree, as shown in Figure 4B. C-index is $0.767(95 \%$ CI: $0.711-0.824)$, indicating that the prediction model has a good discriminability, as shown in Figure 5B.

\section{Decision Curve Analysis of the Prediction Model}

A decision curve analysis (DCA) was applied to assess the clinical validity of the nomogram (Figure 7A and B). The horizontal line means that when all the samples were negative $(\mathrm{Pi}<\mathrm{Pt})$ and no one intervened, the natural net benefit was 
Table 2 Comparison of Laboratory Test Results in the Training Cohort

\begin{tabular}{|c|c|c|c|c|}
\hline Variable & $\begin{array}{c}\text { Non-CI-AKI Group } \\
(n=684)\end{array}$ & $\begin{array}{c}\text { CI-AKI Group } \\
(n=\mid 25)\end{array}$ & $t / Z$ value & $P$ value \\
\hline Triglycerides (mmol/L) & $\mathrm{I} .47(\mathrm{I} .04-2.5 \mathrm{I})$ & $1.32(0.93-2.17)$ & -1.091 & 0.275 \\
\hline Total cholesterol $(\mathrm{mmol} / \mathrm{L})$ & $3.93 \pm|.5|$ & $3.8 I \pm 1.47$ & 1.250 & 0.212 \\
\hline High-density lipoprotein (mmol/L) & $1.19 \pm 0.46$ & $1.14 \pm 0.24$ & 1.867 & 0.062 \\
\hline Low-density lipoprotein $(\mathrm{mmol} / \mathrm{L})$ & $2.64 \pm 1.07$ & $2.7 I \pm 0.82$ & 0.768 & 0.443 \\
\hline Albumin $(g / L)$ & $41.95 \pm 4.68$ & $39.28 \pm 4.87$ & 4.258 & $<0.001$ \\
\hline Fasting plasma glucose (mmol/L) & $5.62(4.97-7.26)$ & $5.86(5.18-7.53)$ & -2.055 & 0.04 \\
\hline Fibrinogen $(g / L)$ & $3.17 \pm 1.01$ & $3.54 \pm 1.20$ & 0.053 & 0.012 \\
\hline FAR & $7.06(5.59-9.12)$ & $9.27(7.58-11.54)$ & -3.819 & $<0.001$ \\
\hline Blood urea $(\mathrm{mmol} / \mathrm{L})$ & $5.97 \pm 2.23$ & $6.04 \pm 2.26$ & -0.332 & 0.740 \\
\hline Serum creatinine $(\mu \mathrm{mol} / \mathrm{L})$ & $70.38 \pm 22.03$ & $71.70 \pm 49.89$ & -0.290 & 0.772 \\
\hline Uric acid $(\mu \mathrm{mol} / \mathrm{L})$ & $320.7 I \pm 95.32$ & $3|5.7| \pm 99.75$ & 0.534 & 0.594 \\
\hline Cystatin C (mg/L) & $0.87 \pm 0.23$ & $0.92 \pm 0.35$ & $-0.88 I$ & 0.383 \\
\hline eGFR (mL/min) & $108.49 \pm 37.08$ & $104.36 \pm 28.00$ & -0.776 & 0.439 \\
\hline Lipoprotein $(\mathrm{A})(\mathrm{mg} / \mathrm{L})$ & $268.13 \pm 190.77$ & $310.26 \pm 223.37$ & -1.665 & 0.098 \\
\hline Total bilirubin $(\mu \mathrm{mol} / \mathrm{L})$ & $14.44 \pm 8.65$ & $14.27 \pm 6.27$ & -0.371 & 0.710 \\
\hline Direct bilirubin $(\mu \mathrm{mol} / \mathrm{L})$ & $4.99 \pm 3.90$ & $4.98 \pm 3.47$ & -0.669 & 0.504 \\
\hline Glycosylated hemoglobin (\%) & $6.90 \pm 1.52$ & $7.06 \pm 1.80$ & -0.782 & 0.435 \\
\hline LVEF (\%) & $58(52-63)$ & $53(50-56.5)$ & -6.393 & $<0.001$ \\
\hline hsCRP (mg/L) & $5.23(1.60,8.60)$ & $7.84(5.65,10.24)$ & -6.834 & $<0.001$ \\
\hline Monocyte count $\left(10^{9} / \mathrm{L}\right)$ & $0.53 \pm 0.65$ & $0.48 \pm 0.69$ & 0.514 & 0.607 \\
\hline Neutrophil count $\left(10^{9} / \mathrm{L}\right)$ & $5.43(3.90-7.44)$ & $5.80(4.57-8.17)$ & -2.472 & 0.013 \\
\hline Lymphocyte count $\left(10^{9} / \mathrm{L}\right)$ & $1.50(1.10-1.90)$ & $1.20(0.90-1.60)$ & -4.133 & $<0.001$ \\
\hline NLR & $3.6 I(2.32-6.22)$ & $5.11(2.96-9.02)$ & -4.223 & $<0.001$ \\
\hline Hemoglobin $(g / L)$ & $140.86 \pm 15.72$ & $131.24 \pm 26.17$ & 2.983 & $<0.001$ \\
\hline Platelet count $\left(10^{9} / \mathrm{L}\right)$ & $197.43 \pm 63.60$ & $185.10 \pm 60.32$ & 1.635 & 0.102 \\
\hline Red blood cell distribution width (\%) & $12.90 \pm 0.80$ & $13.03 \pm 0.97$ & -0.309 & 0.757 \\
\hline Platelet distribution width (\%) & $14.69 \pm 4.84$ & $14.87 \pm 2.56$ & -2.696 & 0.008 \\
\hline
\end{tabular}

Abbreviations: $\mathrm{Cl}-\mathrm{AKI}$, contrast-induced acute kidney injury; FAR, fibrinogen-to-albumin ratio; eGFR, estimated glomerular filtration rate; LVEF, left ventricular ejection fraction; hsCRP, High-sensitive C-reactive protein; NLR, neutrophil-to-lymphocyte ratio.

zero. The grey line represents that all of the samples were positive, and all of the people were intervened. From the decision curves, the nomogram could acquire great net benefits across a large range of high-risk thresholds. From Figure 8, we can see the red curve shows the number of subjects classified as positive (Number high risk) by the nomogram model for each threshold probability; The black curve (Number high risk with event) is the number of true positives under each threshold probability. It means good consistency between the actual distribution and the model's predicted distribution.

\section{Discussion}

Contrast-induced acute kidney injury (CI-AKI) is one of the current complications of undergoing coronary angiography (CAG) or PCI, and CI-AKI increases the length of stay and mortality of patients. ${ }^{3}$ The incidence of CI-AKI has been reported to range from $0 \%$ to $24 \%$. A wide range of studies has been reported due to different definitions, background risk factors, type and dose of contrast agent used, and frequency of other potential causes of coexisting acute renal failure. ${ }^{21}$ Currently, there are no specific clinical therapeutic agents or tools for the development of CI-AKI, and prevention targeting its causative mechanisms and risk factors remains important. Risk assessment of high-risk populations is a primary goal and an important component of CI-AKI prevention. A retrospective analysis of CI-AKI prediction models by Silver et al in 2015 found that risk prediction models are still in their early stages. ${ }^{8}$ The more commonly used Mehran risk score model, which contains eight variables and certain variables that are not readily available preprocedure, limits their use in clinical practice. ${ }^{10}$ 
$\begin{array}{llllllllllllllllllll}20 & 20 & 20 & 20 & 16 & 15 & 13 & 10 & 7 & 6 & 5 & 5 & 4 & 2 & 2 & 2 & 1 & 1 & 1 & 1\end{array}$

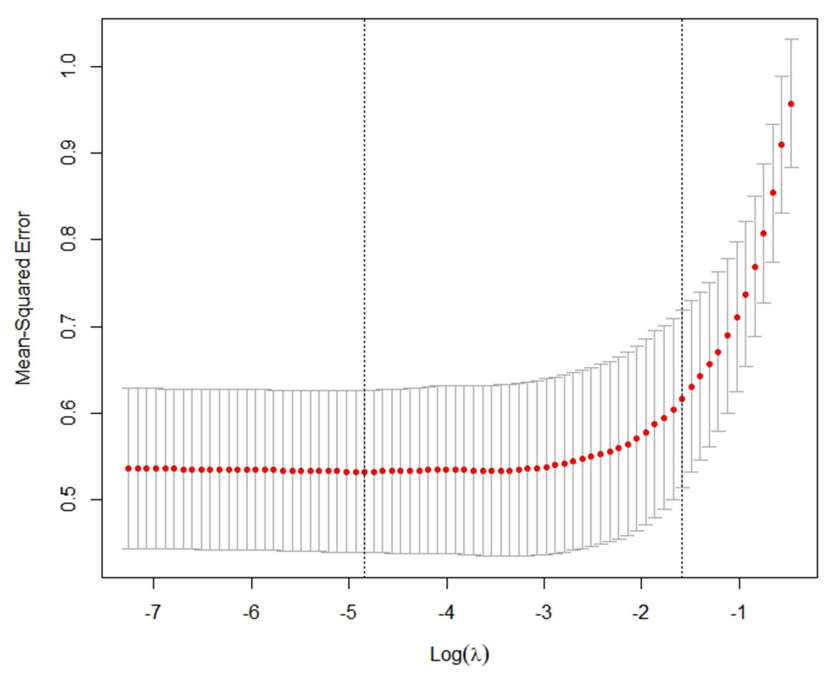

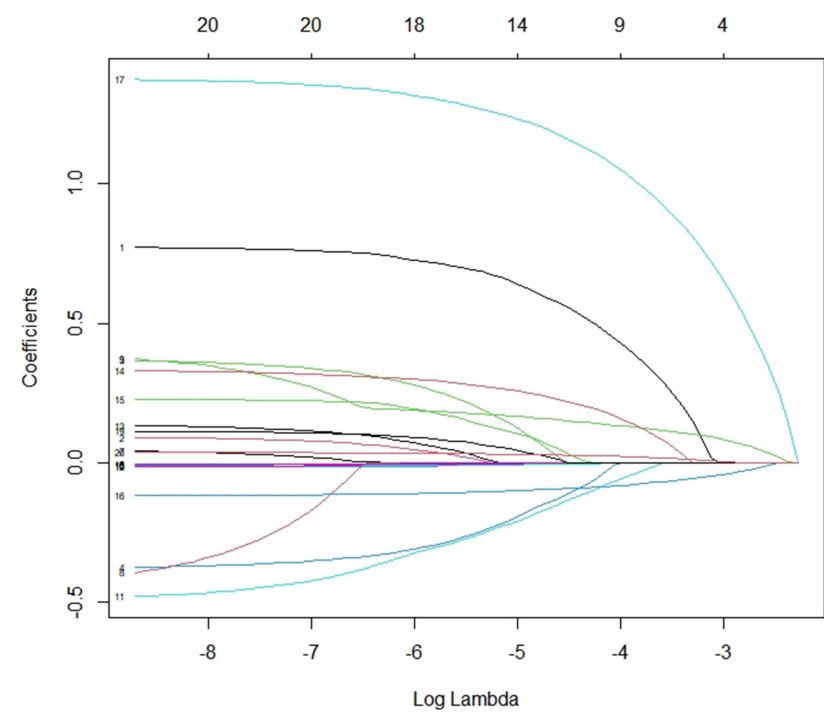

Figure 2 LASSO regression model screening predictors. (A) Vertical lines are plotted at the most available parameter value $\lambda=0.0105$, and the selected variables are 15 ; (B) Plot of each clinical characteristic coefficient against $\log (\lambda)$ by adjusting the parameter $\lambda$.

This study found a $14.5 \%$ (168/1156) incidence of CI-AKI after PCI in patients with NSTE-ACS. We comprehensively screened the independent risk factors for CI-AKI after PCI by retrieving the demographic, medical history, and bio laboratory indices of 1156 NSTE-ACS patients. At the same time, a nomogram prediction model for CI-AKI in NSTEACS patients was developed according to standard procedures. It is important to emphasize that this study focused on easier prediction of the likelihood of CI-AKI after PCI by the nomogram model.

In the results of this study, the prevalence of CI-AKI was significantly higher in the age $>75$ years group than the age $\leq 75(25.85 \%$ vs $13.14 \%, P<0.001)$. The reason may be that increased vascular stiffness and decreased endothelial function with age, which affect vascular function, endothelial gene expression, and monolayer integrity. All these factors together reduce the likelihood of rapid creatinine recovery in postoperative patients and add to the risk of CI-AKI in elderly patients. ${ }^{22,23}$ In contrast, the high glucose status of the diabetic organism may increase the role of reactive oxygen species (ROS) in oxidative damage to the renal parenchyma (especially outer medullary hypoxia) and promote renal injury. ${ }^{24,25}$ Furthermore, CI-AKI may aggravate the pathophysiological process of pre-existing diabetes and diabetic nephropathy, both of which are mutually responsible for the persistent impairment of renal function. In the current risk score, age $>75$ years and diabetes mellitus are risk factors for CI-AKI after elective PCI, which is consistent with our study. ${ }^{26}$ Our data show that patients with low LVEF are more likely to develop CI-AKI. It might be due to the deterioration of cardiac function, where prolonged low cardiac output leaves patients with inadequate tissue and organ perfusion and reduced function, further leading to impaired renal function and poor prognosis. In addition, reduced LVEF is an independent predictor of long-term mortality after cardiac catheterization. ${ }^{27}$ FAR, a novel inflammatory index,

Table 3 Multivariate Logistic Regression Analysis for the Occurrence of $\mathrm{Cl}-\mathrm{AKI}$ After $\mathrm{PCl}$ in Patients with NSTE-ACS in Training Cohort

\begin{tabular}{|l|c|c|c|c|c|}
\hline Variable & $\boldsymbol{\beta}$ & $\mathbf{s} \bar{\chi}$ & Wald $\chi^{2}$ & OR (95\% CI) & $\boldsymbol{P}$ value \\
\hline Age $>75$ & 0.835 & 0.258 & 10.471 & $2.306(1.390-3.824)$ & 0.001 \\
Diabetes mellitus & 1.403 & 0.227 & 38.125 & $4.066(2.605-6.346)$ & $<0.001$ \\
LVEF & -0.112 & 0.020 & 31.253 & $0.894(0.860-0.930)$ & $<0.001$ \\
FAR & 0.186 & 0.035 & 27.631 & $1.205(1.124-1.291)$ & $<0.001$ \\
Lymphocyte count & -0.358 & 0.166 & 4.651 & $0.699(0.505-0.968)$ & 0.031 \\
hsCRP & 0.037 & 0.010 & 14.591 & $1.037(1.018-1.057)$ & $<0.001$ \\
\hline
\end{tabular}

Abbreviations: LVEF, left ventricular ejection fraction; FAR, fibrinogen-to-albumin ratio; hsCRP, hypersensitive $C$ reactive protein. 


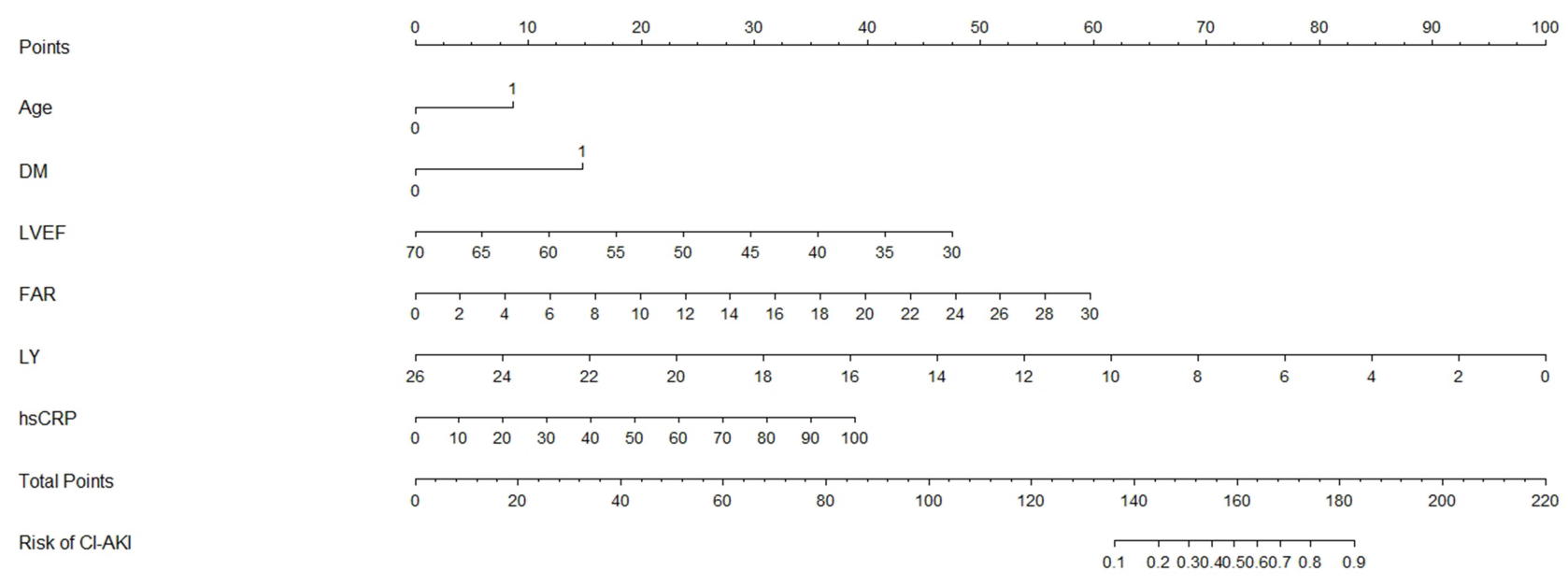

Figure 3 The nomogram for predicting the occurrence of $\mathrm{Cl}-\mathrm{AKI}$ after $\mathrm{PCl}$ in patients with NSTE-ACS. The final score (ie, total points) is calculated as the sum of the individual scores of each of the six variables included in the nomogram.

Abbreviations: $\mathrm{Cl}-\mathrm{AKI}$, contrast-induced acute kidney injury; PCl, percutaneous transluminal coronary intervention; NSTE-ACS, non-ST elevation acute coronary syndrome; DM, diabetes mellitus; LVEF, left ventricular ejection fraction; FAR, fibrinogen-to-albumin ratio; LY, lymphocyte count; hsCRP, High-sensitive C-reactive protein.
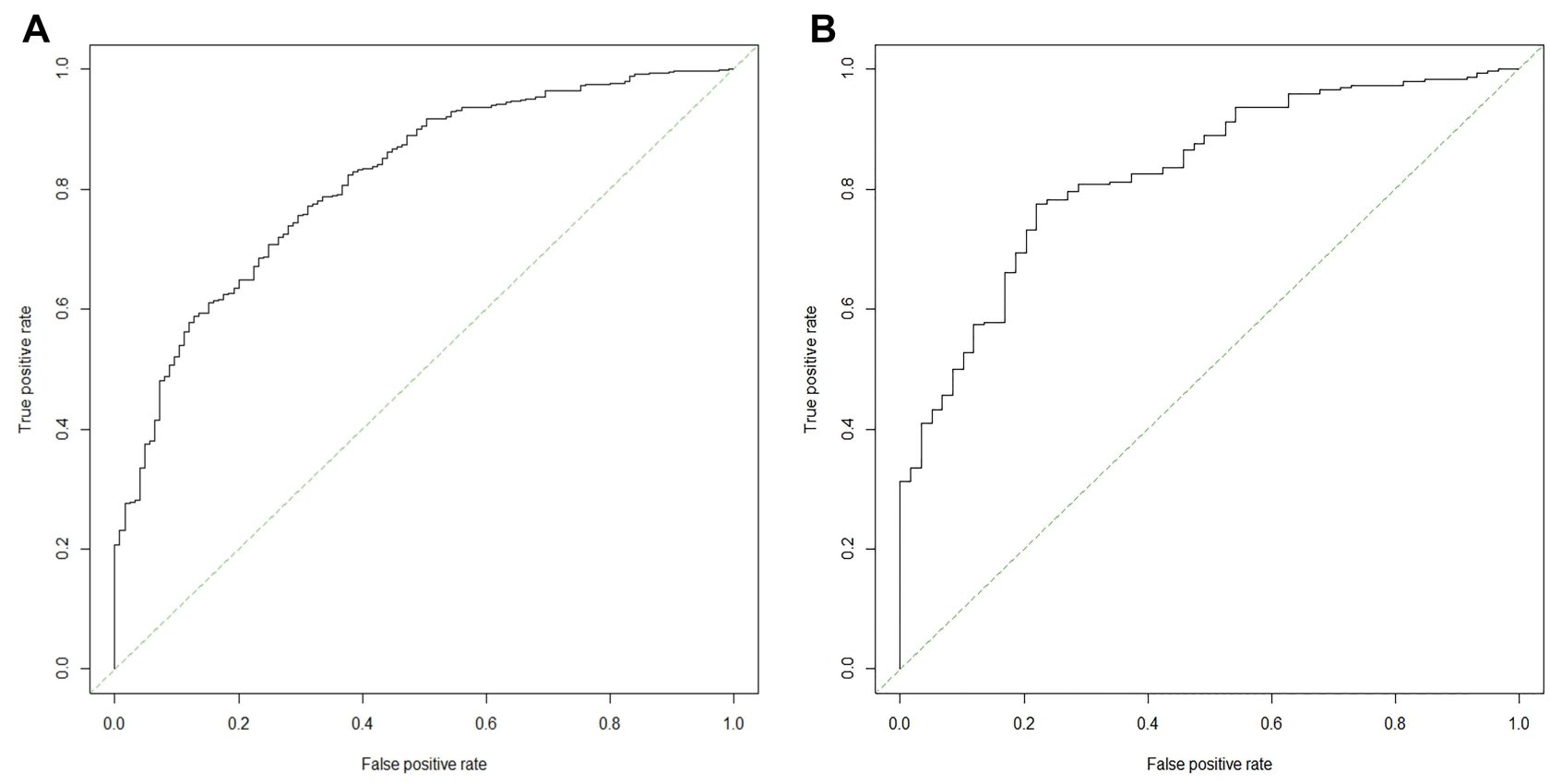

Figure 4 Calibration curve of the nomogram for the training set $(\mathbf{A})$ and the validation set $(\mathbf{B})$. The $\mathrm{X}$-axis represents the overall predicted probability of $\mathrm{Cl}$-AKI after $\mathrm{PCl}$ and the $\mathrm{Y}$-axis represents the actual probability. Model calibration is indicated by the degree of fitting of the curve and the diagonal.

reflects both overactive coagulation and inflammatory pathways, both of which are potential mechanisms for CIAKI. ${ }^{28,29}$ This hypothesis is supported by the correlation between FAR and CRP in previous studies. ${ }^{30}$ Although the exact mechanism of high inflammatory state and the occurrence of CI-AKI is not known. However, it has been shown that the application of statins that improve the inflammatory vascular state can reduce circulating hsCRP, and high-dose statins have been found to reduce the occurrence of CI-AKI. ${ }^{31,32}$ In our study, we also found that lymphocyte counts were statistically significant. Lymphocytes are the core of the immune response and inflammation promotes apoptosis of lymphocytes. The results of Kocas et $\mathrm{al}^{33}$ showed that Platelet-to-Lymphocyte Ratio was an independent predictor of CIAKI after angiography in patients with NSTE-ACS and that lymphocytopenia was associated with the occurrence of 

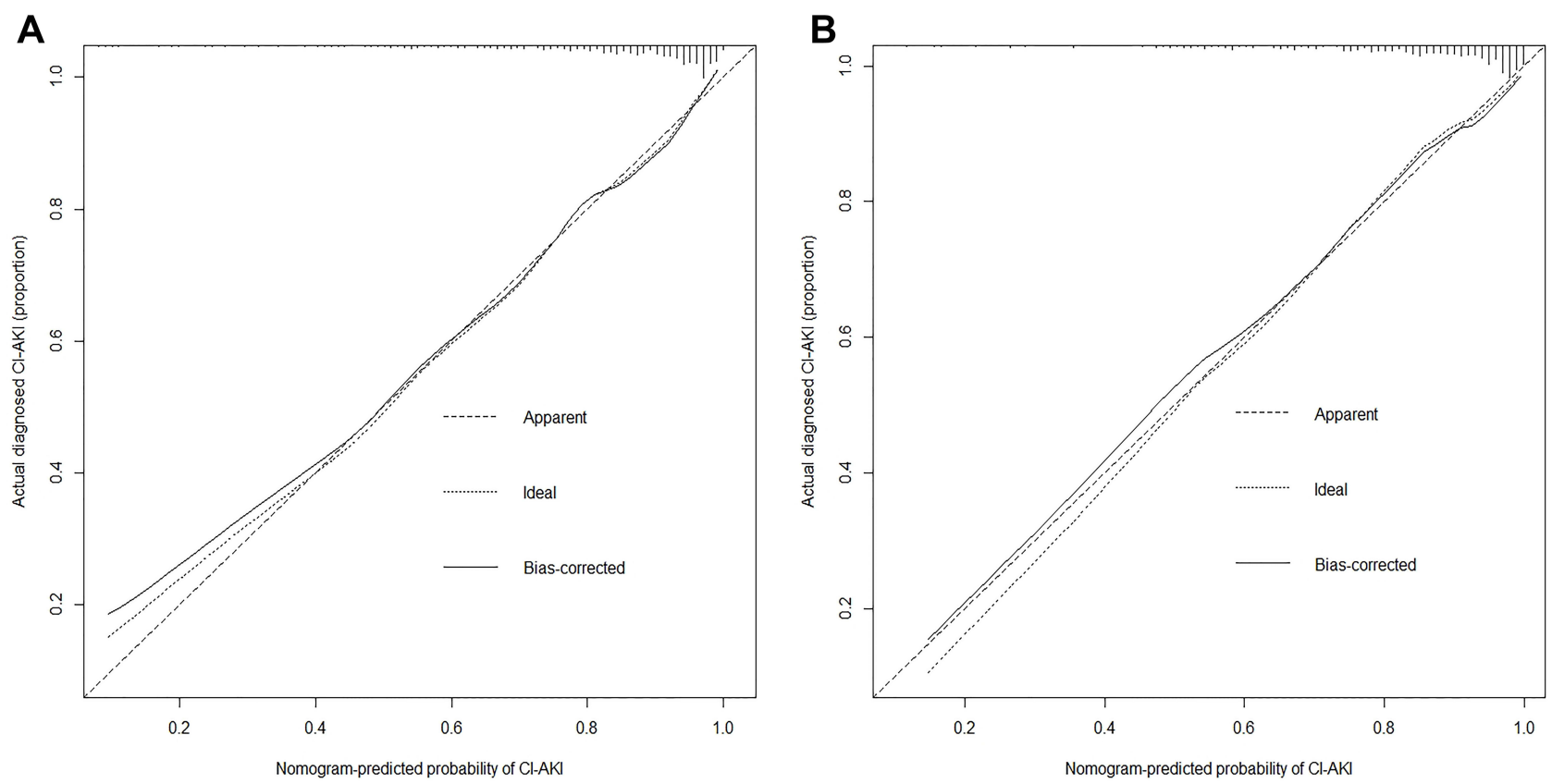

Figure 5 ROC curve of the nomogram for predicting Cl-AKI after PCl in NSTE-ACS patients. (A) ROC curve in the training set; (B) ROC curve in the validation set. Abbreviations: AUC, area under the ROC curve; ROC, receiver operating characteristic; PCI, percutaneous transluminal coronary intervention; NSTE-ACS, non-ST elevation acute coronary syndrome.

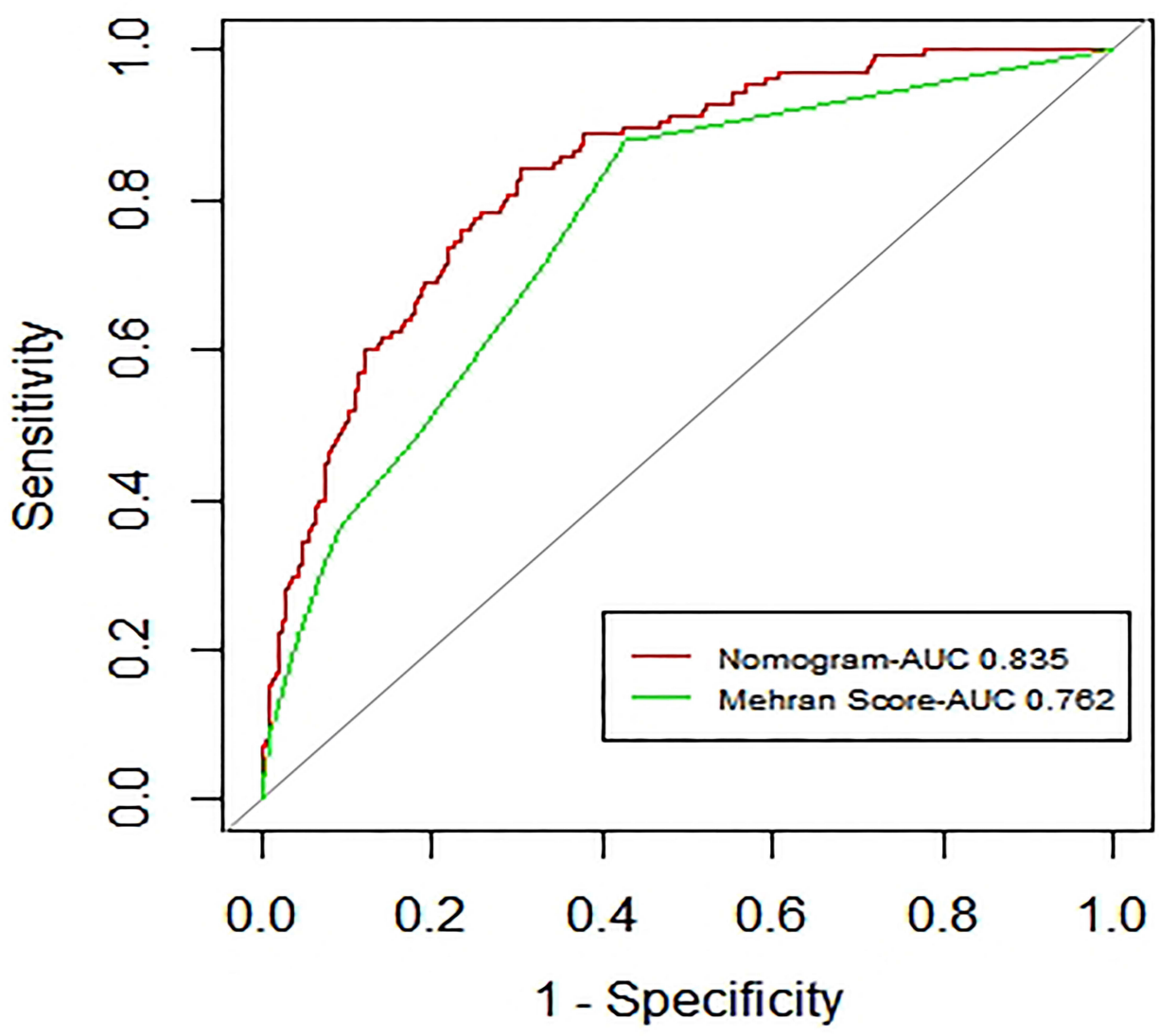

Figure 6 The receiver operator characteristic curves of the nomogram and the Mehran Score. Abbreviation: AUC, area under the curve. 

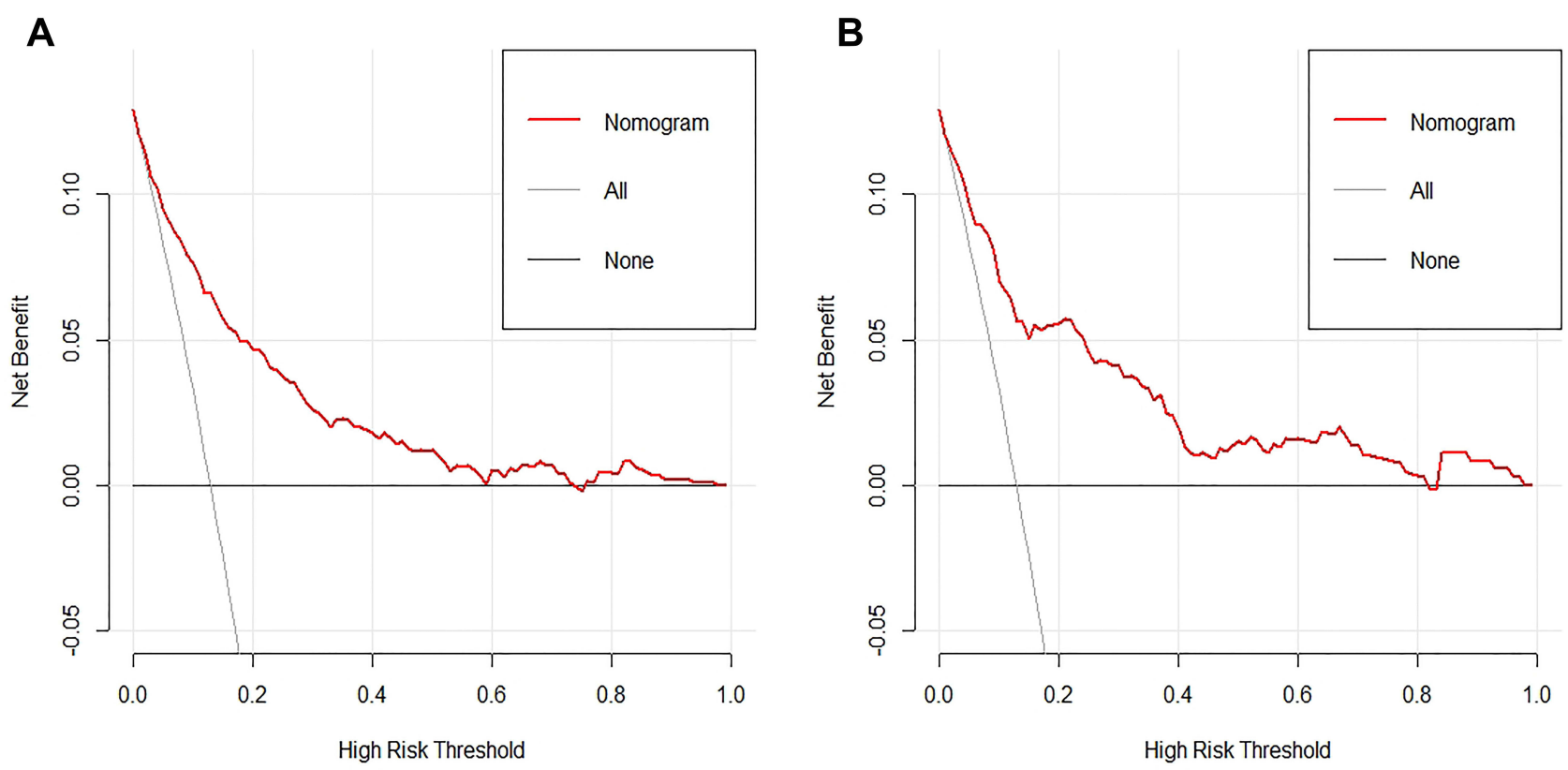

Figure 7 Decision curve analysis for the training set $(\mathbf{A})$ and the validation set $(\mathbf{B})$. A horizontal line indicates that all samples are negative and not treated, with a net benefit of zero. An oblique line indicates that all samples are positive. The net benefit has a negative slope.

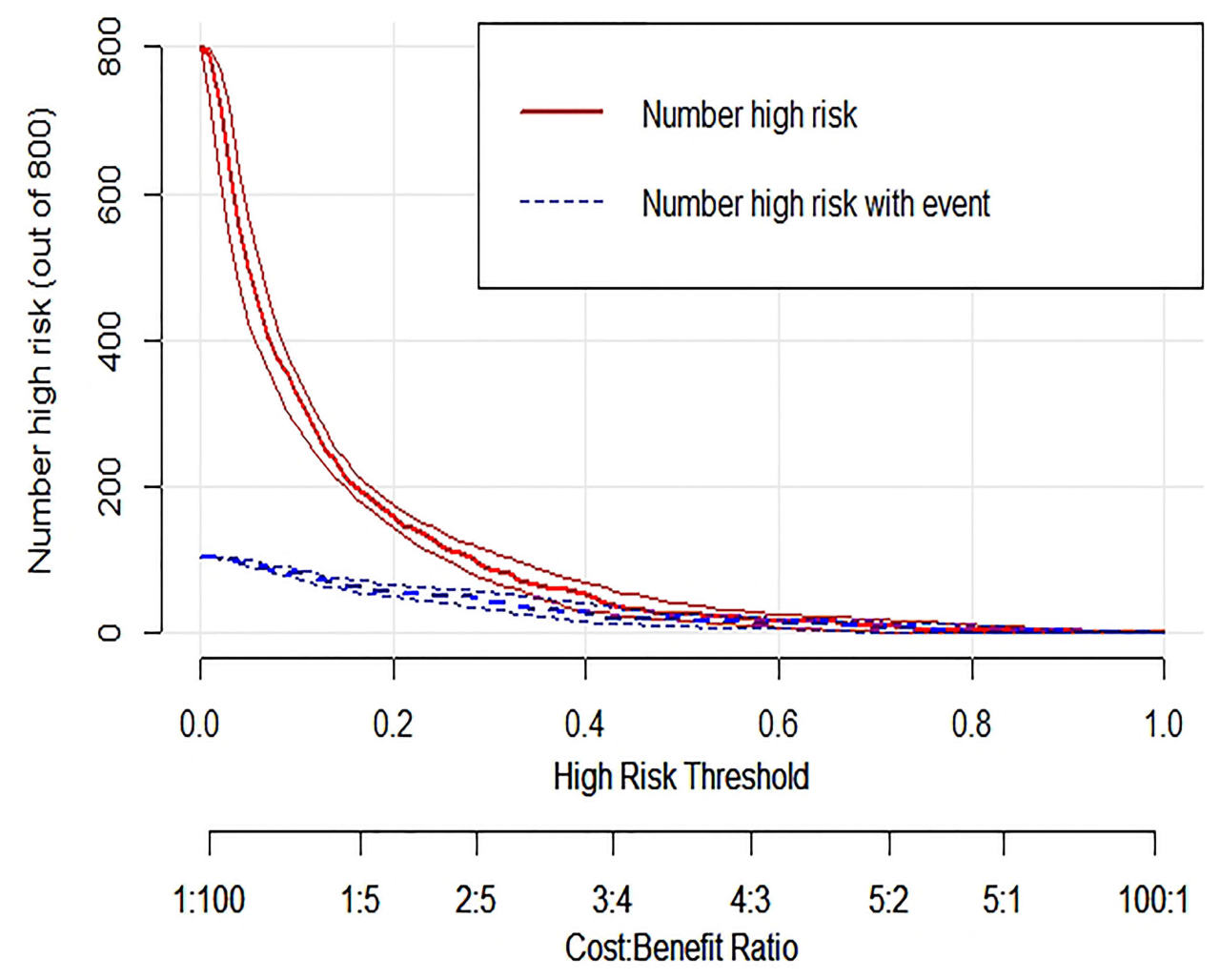

Figure 8 The clinical impact curve of the validation cohort is drawn based on the nomogram. Clinical impact curve of the nomogram plots the number of Cl-AKI patients classified as high risk, and the number of cases classified as high risk with the event at each risk threshold. 
adverse events in patients with coronary artery disease. This is consistent with the results of our study. Therefore, the application of these parameters in the model is more than adequate.

At present, some studies have suggested that female gender, hypotension, anemia, contrast volume, baseline renal function, and other variables are independent risk factors for CI-AKI. ${ }^{34,35}$ Estrogen adversely affects both the reninangiotensin system and renal blood perfusion. ${ }^{36}$ Erythropoietin inhibits apoptosis, promotes renal tubular epithelial cell regeneration, and ameliorates the effects of ischemia and hypoxia-induced renal damage. Therefore, erythropoietin deficiency in anemia individuals may lead to further deterioration of renal function. ${ }^{37}$ However, the present study did not yield similar results, probably since $68 \%$ of the patients in the present study were male, and this significant gender imbalance produced a bias. Simultaneously, Pre-procedure renal function was expected in the NSTE-ACS patients enrolled in our study. Clinicians perform a thorough preprocedural evaluation of patients with high serum creatinine levels. Interventional physicians who were aware of the pre-PCI renal function, apart from reducing the amount of contrast used during the procedure, might have been more aggressive with hydration in patients that could tolerate it. ${ }^{38,39}$ Moreover, the current study concluded that complications and hemodynamic instability are more associated with the development of AKI after contrast exposure than with large amounts of contrast and that reducing the amount of contrast within a certain range does not reduce the risk of CI-AKI. ${ }^{8,40,41}$ In our study cohort, there was no statistically significant relationship between contrast dose and CI-AKI. In addition, this study was a retrospective analysis, there may be differences in the collection of patient history, and we also expect that the factors in the final prediction model are relatively simple and easy to determine.

Nomogram is a visual graph consisting of high and low different lines for predicting the incidence of a clinical event. In this study, we finally included six predictive indicators: "age > 75 years," "diabetes mellitus," "FAR," "LVEF," "hsCRP," and "lymphocyte count" to establish a nomogram. The nomogram has good discriminatory power in the training and validation cohorts. The validity and applicability of the model were demonstrated to some extent, making our risk prediction more clinically attractive. Healthcare professionals can predict the incidence of CI-AKI after PCI based on the summation of scores for each risk factor. In addition to the uncontrollable factors such as age, renal function status, and underlying disease, healthcare professionals should enhance the management of controllable factors, such as adequate hydration therapy (eg, saline, sodium bicarbonate) or combined medications, to actively prevent the occurrence of CI-AKI after PCI.

In summary, our nomogram incorporates six risk factors readily available at patient admission. The strength of our study is that the predictors in the model are routinely tested before PCI, which allows physicians to assess the risk of CI$\mathrm{AKI}$ and take further preoperative preventive measures to reduce the occurrence of CI-AKI by simple calculations before PCI, which has high clinical application and worthy of further use.

\section{Limitations}

There are some limitations of this study. First, this is a single-center retrospective study, and the inherent limitations of such studies inevitably affect the patient selection and produce selection bias. Second, some of the risk factors mentioned in previous studies could not be obtained. Third, the cases in this study were from a single source, and although external validation was available, the data were all from different periods in the same hospital, so multi-center and expanded sample sizes are still needed further to evaluate the clinical predictive value of the model.

\section{Conclusions}

The risk score model developed in this study is a simple and accurate tool. Based on these six laboratories and clinical parameters, clinicians can easily and accurately assess the individual risk of CI-AKI in patients before PCI and make the right clinical decisions to plan and initiate the most appropriate disease management in time.

\section{Ethics Approval and Informed Consent}

The study was conducted in accordance with the Declaration of Helsinki and has been approved by the Medical Ethics Committee of the Affiliated Hospital of Xuzhou Medical University. Due to the study being a retrospective analysis, the review committee waived the requirement for written informed consent. Confidential patient information was removed from the entire data set prior to analysis. 


\section{Author Contributions}

All authors made a significant contribution to the work reported, whether that is in the conception, study design, execution, acquisition of data, analysis and interpretation, or in all these areas; took part in drafting, revising or critically reviewing the article; gave final approval of the version to be published; have agreed on the journal to which the article has been submitted; and agree to be accountable for all aspects of the work.

\section{Funding}

The authors received no financial support for the research, authorship, and/or publication of this article.

\section{Disclosure}

The authors declared no potential conflicts of interest with respect to the research, authorship, and/or publication of this article.

\section{References}

1. Khwaja A. KDIGO clinical practice guidelines for acute kidney injury. Nephron Clin Pract. 2012;120(4):c179-c184. doi:10.1159/000339789

2. Mehran R, Dangas GD, Weisbord SD. Contrast-associated acute kidney injury. N Engl J Med. 2019;380(22):2146-2155. doi:10.1056/ NEJMra1805256

3. McCullough PA, Choi JP, Feghali GA, et al. Contrast-induced acute kidney injury. J Am Coll Cardiol. 2016;68(13):1465-1473. doi:10.1016/j. jacc.2016.05.099

4. Nash K, Hafeez A, Hou S. Hospital-acquired renal insufficiency. Am J Kidney Dis. 2002;39(5):930-936. doi:10.1053/ajkd.2002.32766

5. Yeh RW, Sidney S, Chandra M, Sorel M, Selby JV, Go AS. Population trends in the incidence and outcomes of acute myocardial infarction. $N$ Engl $J$ Med. 2010;362(23):2155-2165. doi:10.1056/NEJMoa0908610

6. Timmis A. Acute coronary syndromes. BMJ. 2015;351:h5153. doi:10.1136/bmj.h5153

7. Chan MY, Sun JL, Newby LK, et al. Long-term mortality of patients undergoing cardiac catheterization for ST-elevation and non-ST-elevation myocardial infarction. Circulation. 2009;119(24):3110-3117. doi:10.1161/CIRCULATIONAHA.108.799981

8. Silver SA, Shah PM, Chertow GM, Harel S, Wald R, Harel Z. Risk prediction models for contrast induced nephropathy: systematic review. BMJ. 2015;351:h4395. doi:10.1136/bmj.h4395

9. Allen DW, Ma B, Leung KC, et al. Risk prediction models for contrast-induced acute kidney injury accompanying cardiac catheterization: systematic review and meta-analysis. Can J Cardiol. 2017;33(6):724-736. doi:10.1016/j.cjca.2017.01.018

10. Mehran R, Aymong ED, Nikolsky E, et al. A simple risk score for prediction of contrast-induced nephropathy after percutaneous coronary intervention: development and initial validation. J Am Coll Cardiol. 2004;44(7):1393-1399. doi:10.1016/j.jacc.2004.06.068

11. Park SY. Nomogram: an analogue tool to deliver digital knowledge. J Thorac Cardiovasc Surg. 2018;155(4):1793. doi:10.1016/j.jtcvs.2017.12.107

12. Xiao S, Zhang L, Wu Q, et al. Development and validation of a risk nomogram model for predicting revascularization after percutaneous coronary intervention in patients with acute coronary syndrome. Clin Interv Aging. 2021;16:1541-1553. doi:10.2147/CIA.S325385

13. Friedman J, Hastie T, Tibshirani R. Regularization paths for generalized linear models via coordinate descent. J Stat Softw. 2010;33(1):1-22. doi:10.18637/jss.v033.i01

14. Collet JP, Thiele H, Barbato E, et al. 2020 ESC guidelines for the management of acute coronary syndromes in patients presenting without persistent ST-segment elevation. Eur Heart J. 2021;42(14):1289-1367. doi:10.1093/eurheartj/ehaa575

15. Zwart B, Ten Berg JM, van 'T Hof AW, et al. Indications for an early invasive strategy in NSTE-ACS patients. Neth Heart J. 2020;28(3):131-135. doi:10.1007/s12471-019-01337-5

16. Nijssen EC, Rennenberg RJ, Nelemans PJ, et al. Prophylactic hydration to protect renal function from intravascular iodinated contrast material in patients at high risk of contrast-induced nephropathy (AMACING): a prospective, randomised, phase 3, controlled, open-label, non-inferiority trial. Lancet. 2017;389(10076):1312-1322. doi:10.1016/S0140-6736(17)30057-0

17. Andreucci M, Solomon R, Tasanarong A. Side effects of radiographic contrast media: pathogenesis, risk factors, and prevention. Biomed Res Int. 2014;2014:741018. doi:10.1155/2014/741018

18. Stacul F, van der Molen AJ, Reimer P, et al. Contrast induced nephropathy: updated ESUR Contrast Media Safety Committee guidelines. Eur Radiol. 2011;21(12):2527-2541. doi:10.1007/s00330-011-2225-0

19. Li M, Tang C, Luo E, Qin Y, Wang D, Yan G. Relation of fibrinogen-to-albumin ratio to severity of coronary artery disease and long-term prognosis in patients with non-ST elevation acute coronary syndrome. Biomed Res Int. 2020;2020:1860268. doi:10.1155/2020/1860268

20. Mehta RL, Kellum JA, Shah SV, et al. Acute Kidney Injury Network: report of an initiative to improve outcomes in acute kidney injury. Crit Care. 2007;11(2):R31. doi:10.1186/cc5713

21. Hossain MA, Costanzo E, Cosentino J, et al. Contrast-induced nephropathy: pathophysiology, risk factors, and prevention. Saudi J Kidney Dis Transpl. 2018;29(1):1-9. doi:10.4103/1319-2442.225199

22. Stojanović SD, Fiedler J, Bauersachs J, Thum T, Sedding DG. Senescence-induced inflammation: an important player and key therapeutic target in atherosclerosis. Eur Heart J. 2020;41(31):2983-2996. doi:10.1093/eurheartj/ehz919

23. Brandes RP, Fleming I, Busse R. Endothelial aging. Cardiovasc Res. 2005;66(2):286-294. doi:10.1016/j.cardiores.2004.12.027

24. Qin YH, Yan GL, Ma CL, Tang CC, Ma GS. Effects of hyperglycaemia and elevated glycosylated haemoglobin on contrast-induced nephropathy after coronary angiography. Exp Ther Med. 2018;16(1):377-383. doi:10.3892/etm.2018.6183

25. Heyman SN, Rosen S, Khamaisi M, Idée JM, Rosenberger C. Reactive oxygen species and the pathogenesis of radiocontrast-induced nephropathy. Invest Radiol. 2010;45(4):188-195. doi:10.1097/RLI.0b013e3181d2eed8 
26. Guo BL, Ouyang FS, Yang SM, et al. Development of a preprocedure nomogram for predicting contrast-induced acute kidney injury after coronary angiography or percutaneous coronary intervention. Oncotarget. 2017;8(43):75087-75093. doi:10.18632/oncotarget.20519

27. Wang K, Li HL, Bei WJ, et al. Association of left ventricular ejection fraction with contrast-induced nephropathy and mortality following coronary angiography or intervention in patients with heart failure. Ther Clin Risk Manag. 2017;13:887-895. doi:10.2147/TCRM.S137654

28. You Z, Guo T, Lin F, et al. Fibrinogen-to-albumin ratio predicts contrast-induced nephropathy in patients after emergency percutaneous coronary intervention. Cardiol Res Pract. 2019;2019:8260583. doi:10.1155/2019/8260583

29. Çetin M, Erdoğan T, Kırış T, et al. Predictive value of fibrinogen-to-albumin ratio in acute coronary syndrome. Herz. 2020;45(Suppl 1):145-151. doi:10.1007/s00059-019-4840-5

30. Gao F, Zhou YJ, Zhu X, Wang ZJ, Yang SW, Shen H. C-reactive protein and the risk of contrast-induced acute kidney injury in patients undergoing percutaneous coronary intervention. Am J Nephrol. 2011;34(3):203-210. doi:10.1159/000329534

31. Asher J, Houston M. Statins and C-reactive protein levels. J Clin Hypertens (Greenwich). 2007;9(8):622-628. doi:10.1111/j.15246175.2007.06639.x

32. Leoncini M, Toso A, Maioli M, Tropeano F, Villani S, Bellandi F. Early high-dose rosuvastatin for contrast-induced nephropathy prevention in acute coronary syndrome: results from the PRATO-ACS study (Protective Effect of Rosuvastatin and Antiplatelet Therapy On contrast-induced acute kidney injury and myocardial damage in patients with Acute Coronary Syndrome). J Am Coll Cardiol. 2014;63(1):71-79. doi:10.1016/j. jacc.2013.04.105

33. Kocas C, Yildiz A, Abaci O, et al. Platelet-to-lymphocyte ratio predicts contrast-induced nephropathy in patients with non-ST-segment elevation acute coronary syndrome. Angiology. 2015;66(10):964-968. doi:10.1177/0003319715578057

34. Tziakas D, Chalikias G, Stakos D, et al. Validation of a new risk score to predict contrast-induced nephropathy after percutaneous coronary intervention. Am J Cardiol. 2014;113(9):1487-1493. doi:10.1016/j.amjcard.2014.02.004

35. Lei L, Xue Y, Guo Z, et al. Nomogram for contrast-induced acute kidney injury in patients with chronic kidney disease undergoing coronary angiography in China: a cohort study. BMJ Open. 2020;10(5):e037256. doi:10.1136/bmjopen-2020-037256

36. Sidhu RB, Brown JR, Robb JF, et al. Interaction of gender and age on post cardiac catheterization contrast-induced acute kidney injury. Am $J$ Cardiol. 2008;102(11):1482-1486. doi:10.1016/j.amjcard.2008.07.037

37. Liang W, Yu CJ, Wang QY, Yu J. Anemia is associated with increased risk of contrast-induced acute kidney injury: a systematic review and metaanalysis. Bioengineered. 2021;12(1):648-661. doi:10.1080/21655979.2021.1883887

38. Karamasis GV, Hampton-Till J, Al-Janabi F, et al. Impact of point-of-care pre-procedure creatinine and eGFR testing in patients with ST segment elevation myocardial infarction undergoing primary PCI: the pilot STATCREAT study. Int J Cardiol. 2017;240:8-13. doi:10.1016/j. ijcard.2017.03.147

39. Bruce RJ, Djamali A, Shinki K, Michel SJ, Fine JP, Pozniak MA. Background fluctuation of kidney function versus contrast-induced nephrotoxicity. AJR Am J Roentgenol. 2009;192(3):711-718. doi:10.2214/AJR.08.1413

40. Rachoin JS, Wolfe Y, Patel S, Cerceo E. Contrast associated nephropathy after intravenous administration: what is the magnitude of the problem. Ren Fail. 2021;43(1):1311-1321. doi:10.1080/0886022X.2021.1978490

41. Hinson JS, Ehmann MR, Fine DM, et al. Risk of acute kidney injury after intravenous contrast media administration. Ann Emerg Med. 2017;69:577-586.e4. doi:10.1016/j.annemergmed.2016.11.021

Clinical Interventions in Aging

Dovepress

\section{Publish your work in this journal}

Clinical Interventions in Aging is an international, peer-reviewed journal focusing on evidence-based reports on the value or lack thereof of treatments intended to prevent or delay the onset of maladaptive correlates of aging in human beings. This journal is indexed on PubMed Central, MedLine, CAS, Scopus and the Elsevier Bibliographic databases. The manuscript management system is completely online and includes a very quick and fair peer-review system, which is all easy to use. Visit http://www.dovepress.com/testimonials.php to read real quotes from published authors.

Submit your manuscript here: https://www.dovepress.com/clinical-interventions-in-aging-journal 